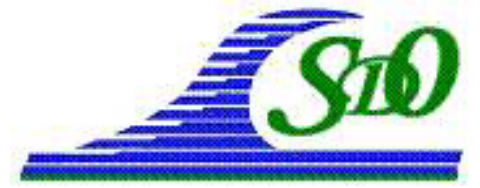

XI ìmes Journées Nationales Génie Côtier - Génie Civil

Les Sables d'Olonne, 22-25 juin 2010

DOI:10.5150/jngcgc.2010.097-S C Editions Paralia CFL

disponible en ligne - http://www.paralia.fr - available online

\title{
Etude de la durabilité de sédiments marins traités avec différents types de fumée de silice
}

\author{
Ernesto SILITONGA ${ }^{1}$, Salim MEZAZIGH ${ }^{1}$, Daniel LEVACHER ${ }^{1}$
}

1. Université de Caen, U.M.R., CNRS 6143 M2C,

24 rue des Tilleuls 14000, Caen, France.

ernesto.silitonga@.unicaen.fr ; salim.mezazigh@unicaen.fr ;

daniel.levacher@unicaen.fr

\section{Résumé :}

L'objectif de cette étude a consisté, à partir de sédiments marins dragués dans le port de Port-en-Bessin, à élaborer un matériau routier susceptible d'être employé pour la réalisation d'une couche de forme. L'utilisation de fumée de silice permet pour les matériaux fins, généralement sensibles à l'eau, la création de liens entre les particules. Ceci a pour effet d'augmenter les résistances mécaniques et de limiter la circulation de l'eau libre dans la structure. Les résultats ont montré en laboratoire que l'effet de fumée de silice sur le comportement mécanique dépend de sa granulométrie et de sa minéralogie. L'effet de l'addition de fumée de silice dans les mélanges à base de sédiments a porté sur le compactage, sur l'évolution des performances mécaniques en compression simple et dans des conditions normales et extrêmes. L'ensemble des résultats acquis lors de ces travaux sont analysés et discutés.

Mots-clés :

Sédiments marins - Valorisation - Fumée de silice - Matériau routier - Compression simple - Essai de mouillage-séchage

\section{Introduction}

Lorsque la zone dragage est à proximité de sites industriels révélant une pollution éventuelle, les sédiments peuvent s'avérer fortement contaminés. Parmi les substances chimiques émises dans l'environnement, certaines s'accumuleront ainsi dans les sédiments fluviaux, estuariens et littoraux. Les substances néfastes les plus souvent citées sont des composés organiques et des métaux lourds à caractère dangereux ou non. Tous ces polluants constituent un problème majeur dans la gestion durable des sédiments. La difficulté de gérer ces sédiments pollués est non seulement au niveau de leur stockage mais aussi dans leur devenir et leur élimination. La recherche de filières de valorisation s'impose comme celles qui utilisent les liants hydrauliques. L'objectif est de stabiliser et de solidifier les sédiments dragués. La stabilisation est obtenue par divers mécanismes : $(i)$ piégeage mécanique, les contaminants métalliques peuvent agir comme centre de nucléation et rester piégés dans les hydrates ainsi formés (COCKE \& MOLLAH, 1992; 1993), (ii) précipitation, la matrice cimentaire étant un milieu 
fortement alcalin, elle favorise la précipitation d'hydroxydes métalliques (COCKE et al., 1989), (iii) adsorption, les hydrates de ciment possèdent une bonne capacité d'adsorption, et particulièrement les C-S-H (COCKE et al., 1989). Notamment la chaux peut être employée avec d'autres additifs ou seule, pour son effet alcalin. L'utilisation de chaux est très efficace dans les sols fortement contaminés. Elle permet de conserver des $\mathrm{pH}$ de 9 à 11 au sein de la matrice sédimentaire, de minimiser la solubilité des métaux présents dans celle-ci, et d'éviter à nouveau leur dissolution (GARCIA et al., 2004). L'évolution de la consistance des sédiments débute dès l'épandage de la chaux avec la fragmentation due au retrait de déshydratation. Le malaxage amplifie ensuite le phénomène. Les travaux de recherche menés par ASAVAPISIT (ASAVAPISIT et al., 2001) montrent que les valeurs de résistance en compression simple les plus élevées sont obtenues avec des mélanges préparés avec $10 \%$ de fumée de silice. Cependant elles diminuent avec un dosage de $20 \%$. Pour un tel dosage, il est possible que l'excès de fumée de silice ait occupé les espaces des pores réduisant ainsi les espaces vides nécessaires pour former les produits résultant de la réaction d'hydratation du ciment. En effet, pour que s'opère le développement des produits d'hydratation du ciment, on a besoin d'espaces vides et l'excès de fumée de silice peut les limiter. Par ailleurs, il a été constaté par ASAVAPISIT et al. (2001) que la fumée de silice a un effet significatif sur la durée de la prise du ciment et l'hydroxyde de plomb. Il a été observé également qu'avec l'augmentation de la quantité de fumée de silice, la durée de prise des échantillons (ciment + hydroxyde de plomb) diminue. Il a été conclu que la production de $\mathrm{C}-\mathrm{S}-\mathrm{H}$ secondaire due à la réaction pouzzolanique obtenue dans la réaction de la fumée de silice avec l'élément $\mathrm{Ca}(\mathrm{OH})_{2}$, entraîne une création de pores isolés et fins ce qui provoque une augmentation de la résistance de diffusion des matériaux alcalins (ASAVAPISIT et al., 2001). BAGHERPOUR \& CHOOBBASTI (2003), ont observé que la présence de fumée de silice dans le traitement de sols fins au ciment et à la chaux, améliore les performances mécaniques des sols ainsi traités. Le sol fin étudié a été traité avec 4 pour cent de chaux et 8 pour cent de ciment Portland. L'ajout en fumée de silice variait de 0 à 5\%. BEHMANESH (2008) a montré que la présence de fumée de silice diminue la porosité. Dans des mélanges traités avec un dosage en ciment de $4 \%$ de ciment et sans ajout de fumée de silice, la perméabilité est d'environ deux fois plus importante que dans le cas de mélanges identiques traités avec seulement $1 \%$ de fumée de silice. Ceci peut être relié à la taille très fine des grains de la fumée de silice utilisée qui permet ainsi de remplir les espaces vides entre les grains dans les matériaux fins solidifiés. En effet, grâce à la fine structure de la fumée de silice, la cristallisation obtenue par la réaction permet de remplir les vides et par voie de conséquence une réduction de la perméabilité dans des échantillons ainsi traités à matrice sédimentaire (BEHMANESH 2008). 


\section{Caractérisation des matériaux}

Les sédiments utilisés dans cette étude proviennent du dragage de bassins de Port-enBessin, en région de Basse-Normandie. Les sédiments ont été traités avec un ajout de trois fumées de silice différentes.

\subsection{Caractéristiques physiques}

L'analyse granulométrique permet d'observer la répartition dimensionnelle des particules constituant tout matériau fin. Une analyse granulométrique a d'abord été effectuée sur des sédiments prélevés sur le site du Port-en-Bessin en 4 points différents, dénommés $\mathrm{A}, \mathrm{B}, \mathrm{C}, \mathrm{D}$. Le tableau 1 détaille la distribution granulométrique obtenue sur les sédiments de Port-en-Bessin pour les 4 points de prélèvement. On constate que cette distribution est similaire pour les 4 points de prélèvement ce qui confirme l'homogénéité des sédiments prélevés à Port-en-Bessin. Cette homogénéité est également observée sur les fractions minérales données dans le même tableau.

Tableau 1. Données granulométriques des sédiments de Port-en-Bessin.

\begin{tabular}{lllll}
\hline & PEB $\boldsymbol{A}-\mathbf{1}$ & $\boldsymbol{P E B} \boldsymbol{B}-\boldsymbol{1}$ & $\boldsymbol{P E B} \boldsymbol{C}-\boldsymbol{1}$ & $\boldsymbol{P E B} \boldsymbol{D}-\boldsymbol{1}$ \\
\hline D10 $(\mu \mathrm{m})$ & 2 & 1.92 & 1.5 & 2.2 \\
D50 $(\mu \mathrm{m})$ & 12 & 11.1 & 10.6 & 12.5 \\
D90 $(\mu \mathrm{m})$ & 78.4 & 66.7 & 72 & 75.6 \\
Fraction argileuse $(<2 \mu \mathrm{m})(\%)$ & 10.7 & 10.6 & 13.8 & 9 \\
Fraction silteuse $(2$ à $63 \mu \mathrm{m})(\%)$ & 77.2 & 78.8 & 74.8 & 78.8 \\
Fraction sableuse $(>63 \mu \mathrm{m})(\%)$ & 12.6 & 10.6 & 11.5 & 12.2 \\
\hline
\end{tabular}

Selon ces résultats, nous pouvons dire que les sédiments de Port-en- Bessin sont majoritairement silteux.

Un des ajouts concerne la fumée de silice dont la finesse est la caractéristique essentielle de la réaction pouzzolanique. Cette réaction permet aux particules des fumées de silice d'être absorbées par celles du ciment et d'empêcher celui-ci de floculer. De plus, les micro-particules de fumée de silice peuvent diminuer l'espace vide entre les particules $\mathrm{du}$ ciment. Ceci provoque ainsi la diminution du rapport $\mathrm{E} / \mathrm{C}$ du mélange $(\mathrm{E}$, masse d'eau et $\mathrm{C}$, masse de liants, à savoir, l'ensemble fumée de silice, chaux et ciment).

En effet, moins il y a d'espaces vides dans le mélange, moins le mélange a besoin d'eau. Ceci contribue à faire chuter le rapport $\mathrm{E} / \mathrm{C}$ du mélange. Les particules fines du ciment ont également la capacité de combler les espaces vides, mais elles se dissolvent rapidement dans l'eau, ce qui n'est pas efficace pour faire du remplissage. Notons qu'avec un faible rapport $\mathrm{E} / \mathrm{C}$, on peut atténuer phénomène de gonflement/expansion. Par contre, avec un rapport élevé de $\mathrm{E} / \mathrm{C}$, le mélange a plus d'espaces disponibles ce qui permet l'hydratation du milieu poreux et la formation de nombreux cristaux 
d'hydroxyde de calcium et d'ettringite, composés responsables du phénomène de gonflement/expansion. La figure 1 rassemble les granulométries des 3 différentes fumées de silice, et des liants (chaux et ciment) utilisés dans cette étude. Le matériau le plus fin est la chaux, puis le ciment jusqu'au diamètre $10 \mu \mathrm{m}$ et la fumée de silice de type 1 et de type 2 . Parmi les 3 fumées de silice retenues, la fumée de silice de type 2 (FS2) contient des particules dont le diamètre est le plus élevé $(200 \mu \mathrm{m})$. Dès lors, nous pouvons considérer que la fumée de silice de type 2 (FS2) sera la moins réactive. Comme expliqué auparavant, la réactivité d'une fumée de silice dépend de sa finesse et de ses caractéristiques chimiques pour diminuer les espaces vides entre les particules de ciment afin de fournir une résistance additionnelle. En revanche, vis-à-vis de la finesse des particules, la fumée de silice de type 1 est la plus réactive. Ceci sera corroboré par la suite avec les essais mécaniques (compression simple) opérés sur les mélanges.

Tableau 2. Granulométries des ajouts et liants : fumées de silice, chaux et ciment.

\begin{tabular}{llllll}
\hline & Chaux & Ciment & FS1 & FS2 & FS3 \\
\hline D10 $(\mu \mathrm{m})$ & 0.5 & 0.6 & 4.0 & 18.2 & 6 \\
D50 $(\mu \mathrm{m})$ & 11.3 & 32.6 & 32.3 & 153 & 49.7 \\
D90 $(\mu \mathrm{m})$ & 172 & 730 & 93 & 307 & 118 \\
Fraction argileuse $(<2 \mu \mathrm{m})(\%)$ & 18.3 & 15.2 & 7.3 & 2.3 & 4.3 \\
Fraction silteuse $(2 \grave{\text { à }} 63 \mu \mathrm{m})(\%)$ & 63.1 & 53.3 & 70.0 & 18.9 & 55.5 \\
Fraction sableuse $(>63 \mu \mathrm{m})(\%)$ & 19.7 & 31.5 & 22.7 & 78.8 & 40.1 \\
\hline
\end{tabular}

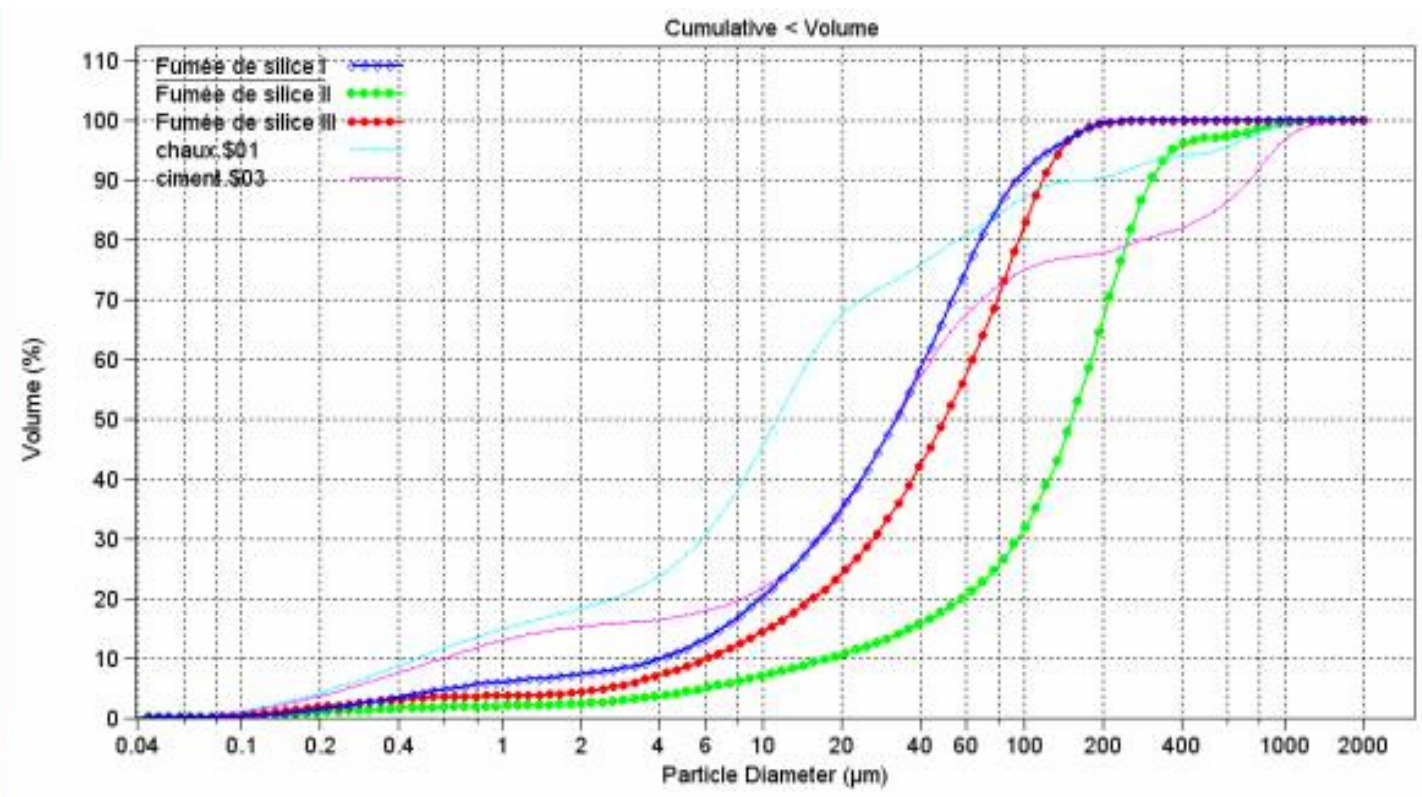

Figure 1. Courbes granulométriques des fumées de silice, de la chaux et du ciment. 


\section{XI $I^{\text {èmes }}$ Journées Nationales Génie Côtier - Génie Civil \\ Les Sables d'Olonne, 22-25 juin 2010}

\subsection{Caractéristiques chimiques}

Les caractéristiques des trois types de fumée de silice retenues sont présentées sur le tableau 3. Les résultats montrent que la fumée de silice de type 1 (FS1) contient un pourcentage de dioxyde de silice $\left(\mathrm{SiO}_{2}\right)$ plus important.

Tableau 3. Composition chimique des trois fumées de silice utilisées.

\begin{tabular}{llll}
\hline & FS $\mathbf{1}$ & $\boldsymbol{F S} \mathbf{2}$ & $\boldsymbol{F S} \mathbf{3}$ \\
\hline Couleur & Grise claire & Bleue & Grise foncée \\
Dioxyde de silice $\left(\mathrm{SiO}_{2}\right)$ & $90-92$ & $85-90$ & $90-95$ \\
Oxyde de fer $\left(\mathrm{Fe}_{2} \mathrm{O}_{3}\right)$ & $1,5-2$ & $1,5-2$ & $1,5-2$ \\
Oxyde d'aluminium $(\mathrm{AI2O})$ & 1 & 1 & $1-1,5$ \\
Oxyde de calcium $(\mathrm{CaO})$ & $0,5-1$ & $0,5-1$ & $0,5-1$ \\
Oxyde de magnésium $(\mathrm{MgO})$ & $1-1,5$ & $1-1,5$ & 1 \\
Oxyde de sodium $\left(\mathrm{Na}_{2} \mathrm{O}\right)$ & $0,5-1$ & $1-1,5$ & $0,8-1$ \\
Oxyde de potassium $\left(\mathrm{K}_{2} \mathrm{O}\right)$ & $1-1,3$ & $1-1,3$ & $1,3-1,5$ \\
Carbone $(\mathrm{C})$ & $0,5-1$ & $1-1,5$ & $1-1,5$ \\
Si libre $(\%)$ & $<0,2$ & $<0,4$ & $<0,4$ \\
CaO libre $(\%)$ & $<1$ & $<2$ & $<1$ \\
SO & $<\%)$ & $<2$ & $<1$ \\
Cl $(\%)$ & $<0,2$ & $<0,5$ & $<0,1$ \\
Surface spécifique $\left(\mathrm{m}^{2} / \mathrm{g}\right)$ & $18-25$ & $15-30$ & $15-35$ \\
Masse volumique $\left(\mathrm{kg} / \mathrm{m}^{3}\right)$ & $500-700$ & $500-700$ & $500-650$ \\
\hline
\end{tabular}

Le fort pourcentage de $\mathrm{SiO}_{2}$ est un facteur important dans l'évolution des performances mécaniques des échantillons. En effet, le $\mathrm{SiO}_{2}$ contribue à la production du silicate calcique hydraté C-S-H. Ce produit une fois hydraté renforce les liaisons entre les particules des sédiments ce qui leur procure une résistance plus élevée. La fumée de silice de type 2 (FS2) présente un pourcentage de $\mathrm{SiO}_{2}$ inférieur aux autres. Pour situer la réactivité de ces fumées, des échantillons ont été préparés uniquement à base de liants. Ceci a permis de visualiser les réactions intervenant entre chaque constituant et ainsi de fixer des références qui permettront par la suite de mener des comparaisons pour chaque mélange de sédiments. En effet, grâce à la fourniture de la silice et de l'alumine par les minéraux argileux contenus dans les sols fins, les réactions pouzzolaniques peuvent durer plusieurs années et contribuent ainsi à améliorer, à long terme, la résistance des ouvrages en créant des produits aux capacités liantes telle que la silicate calcique hydraté (C-S-H) et l'aluminate calcique hydraté (C-A-H). Ainsi, on peut dire que la fumée de silice de type 1 (FS1) contribuera de façon plus importante à l'augmentation des performances mécaniques des sédiments traités par rapport aux autres liants. La fumée de silice de type 2 (FS2) possède un pourcentage de chaux libre et de $\mathrm{CaO}$ plus important. La teneur en chaux libre/CaO est également un paramètre important pour assurer la réussite de la réaction pouzzolanique des liants utilisés. Par 
ailleurs, on constate également que la fumée de silice de type 2 (FS2) contient un pourcentage de $\mathrm{SO}_{3}$ plus élevé que les autres fumées de silice. Ceci peut provoquer une production d'ettringite, facteur jouant un rôle important dans le gonflement et la diminution de la résistance des mélanges.

\section{Méthodes de préparation des échantillons}

La confection des éprouvettes et la préparation des mélanges sédiments-produits de traitement se déroule de la façon suivante. Tout d'abord, les sédiments disposés dans des bacs, subissent un pré-séchage en étuve. La température est fixée à $50^{\circ} \mathrm{C}$ afin d'éviter toute modification des caractéristiques physico-chimiques des sédiments. Au fur et à mesure de leur assèchement, les sédiments apparaissent sous forme de mottes qu'il est nécessaire de fragmenter régulièrement pour permettre un séchage homogène. Par la suite, les sédiments séchés sont fragmentés et passés au tamis de $2 \mathrm{~mm}$. Pour satisfaire aux conditions de réalisation des matériaux stabilisés, les échantillons subissent un compactage pour une énergie de type Proctor Normal, énergie la plus représentative du compactage des sols naturels stabilisés. Le protocole de ce compactage est proposé par LEMEE (2006). Les éprouvettes d'étude ont été réalisées dans des moules cylindriques en plastique de diamètre $40 \mathrm{~mm}$ et de hauteur $80 \mathrm{~mm}$.

\subsection{Dosages des mélanges}

Les masses des différents liants à ajouter sont prélablement déterminées. Les dosages sont calculés par rapport à la masse totale des constituants secs (y compris les liants). Après la détermination des différentes masses en produits de traitement, les sédiments sont placés dans un malaxeur pour obtenir un mélange homogène. La chaux est ensuite ajoutée en cours de malaxage avant d'incorporer les autres liants. Un délai d'une heure est observé. Ceci permet à la chaux d'agir en assurant la floculation des argiles. Pour conserver l'état hydrique du mélange sédiments-chaux, le matériau est retiré du malaxeur et conservé dans un bac surmonté d'un chiffon humide. Le mélange est ensuite reversé dans le malaxeur pour l'ajout des autres liants dont l'opération de malaxage est identique à celle de la chaux. Il a été réalisé treize formulations de mélange. Pour chaque série de mélange, trois éprouvettes ont été faites et ce pour chaque période d'essai soit 7, 14, 28, 60, 90, 180 et 360 jours. Le tableau 4 rassemble les pourcentages de liants utilisés pour les formulations retenues.

\subsection{Analyse des résultats d'essais de résistance en compression simple}

\subsubsection{Mélange sédiments-liants sans incorporation de sable}

Les résultats obtenus pour le sédiment traité sans addition de sable avec les trois types de fumée de silice sont donnés sur la figure 2. Nous remarquons évidemment que les résistances à la compression simple Rc aux jeunes âges ( 7 et 14 jours) pour les 
mélanges traités sont supérieures à celles du mélange de sédiment non traité (A1, A2 et A3 sur la figure 2). Nous observons qu'à ces âges, les résistances des mélanges traités avec la fumée de silice de type 3 (FS3) et de type 1 (FS1) ne montrent pas de différence importante (voir A3). Le mélange traité avec de la fumée de silice de type 2 (FS2) offre une valeur de résistance inférieure à celles des mélanges avec FS1 et FS3 (voir A2).

Tableau 4. Pourcentages de liants retenus dans les mélanges.

\begin{tabular}{lllllll}
\hline Série & $\begin{array}{l}\text { Sable } \\
(\%)\end{array}$ & $\begin{array}{l}\text { Ciment } \\
(\%)\end{array}$ & $\begin{array}{l}\text { Chaux C2 } \\
(\%)\end{array}$ & $\begin{array}{l}\text { Fumée de } \\
\text { silice 1 (\%) }\end{array}$ & $\begin{array}{l}\text { Fumée de } \\
\text { silice 2 (\%) }\end{array}$ & $\begin{array}{l}\text { Fumée de } \\
\text { silice 3 (\%) }\end{array}$ \\
\hline Sédiment non traité & - & - & - & - & - & - \\
$0-2-3 C 2-1 F S 1 *$ & - & 2 & 3 & 1 & - & - \\
$0-2-3 C 2-1 F S 2 *$ & - & 2 & 3 & - & 1 & - \\
$0-2-3 C 2-1 F S 3 *$ & - & 2 & 3 & - & - & 1 \\
$5-2-3 C 2-1 F S 1^{*}$ & 5 & 2 & 3 & 1 & - & - \\
$5-2-3 C 2-1 F S 2 *$ & 5 & 2 & 3 & - & 1 & - \\
$5-2-3 C 2-1 F S 3 *$ & 5 & 2 & 3 & - & - & 1 \\
$15-2-3 C 2-1 F S 1^{*}$ & 15 & 2 & 3 & 1 & - & - \\
$15-2-3 C 2-1 F S 2 *$ & 15 & 2 & 3 & - & 1 & - \\
$15-2-3 C 2-1 F S 3 *$ & 15 & 2 & 3 & - & - & 1 \\
\hline
\end{tabular}

* Les chiffres sont les \% de liants dans l'ordre : sable, ciment, chaux C2 et fumées de silice FS.

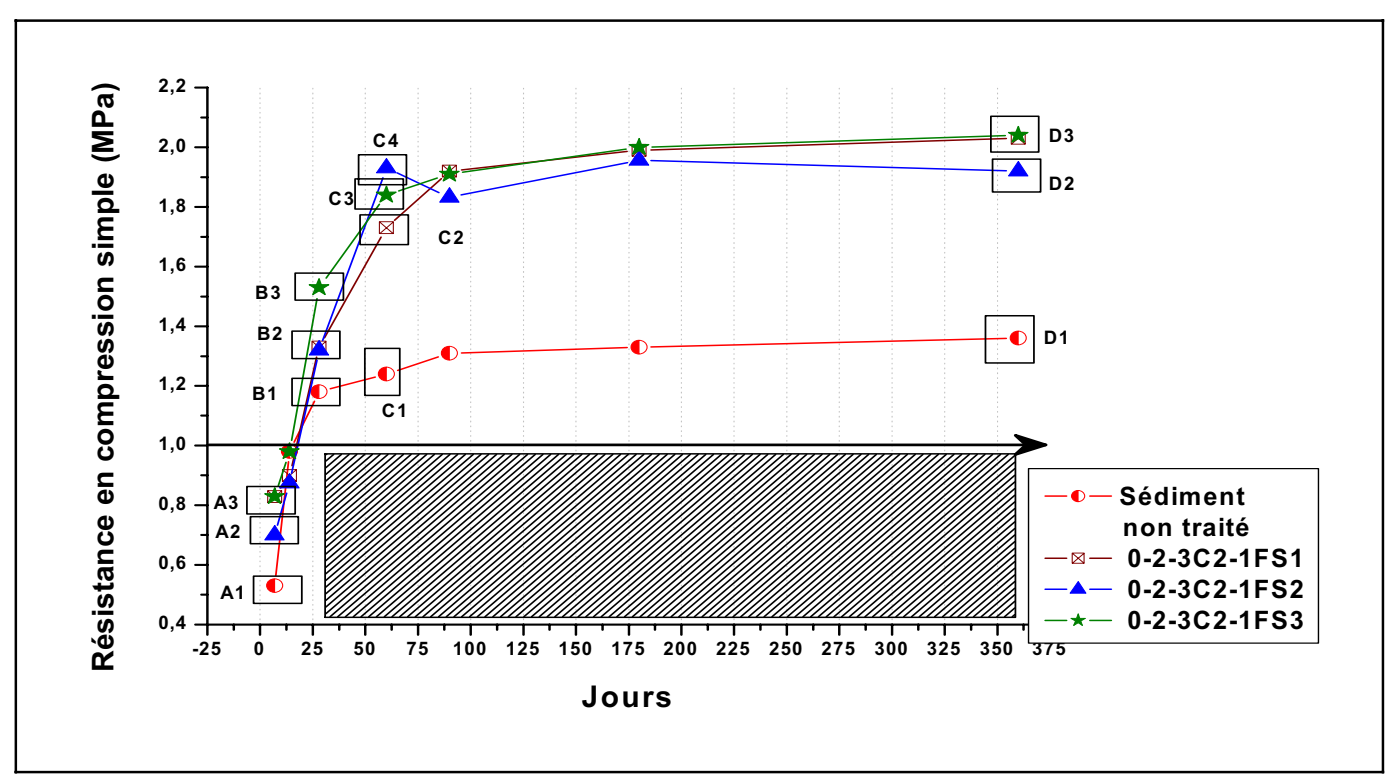

Figure 2. Evolution de Rc pour le sédiment traité avec différents types de fumée de silice sans sable.

L'évolution de la résistance à long terme, de 180 à 360 jours, des mélanges traités avec FS1 et FS3 montre une légère augmentation (voir D3). D'après les résultats obtenus, 
nous pouvons constater que la différence au niveau des caractéristiques des fumées de silices provoque des différences au niveau des résistances mécaniques obtenues. Théoriquement, grâce à ses particules fines, la fumée de silice occupe les espaces des pores et réduit une partie des espaces vides qui n'ont pas été remplis par le produit des hydrations du ciment. Mais cet effet va se montrer plus important quand l'âge des éprouvettes augmente. En effet, avec l'âge des éprouvettes, la réaction pouzzolanique survient. Donc, selon la granulométrie ou la finesse des particules, le mélange traité avec la fumée de silice de type 1 (FS1) devrait en principe fournir la résistance supérieure aux autres. Mais les résultats obtenus montrent que le mélange avec la fumée de silice type 3 (FS3) possède une meilleure résistance que celui traité avec la fumée de silice de type 1 (FS1). On peut suggérer que ce sont alors les caractéristiques chimiques de la fumée de silice qui seraient alors à l'origine de cette augmentation de résistance.

\subsubsection{Essais de compression simple avec une incorporation de 5\% de sable}

Nous observons que les résistances en compression simple obtenues pour tous les mélanges avec un ajout de $5 \%$ de sable, après 28 jours sont supérieures à $1 \mathrm{MPa}$, ce qui signifie que le mélange utilisé serait satisfaisant pour être appliqué en travaux routiers. Les résultats montent également que l'augmentation de la résistance du mélange traité avec FS3 est plus importante que celles des autres mélanges.

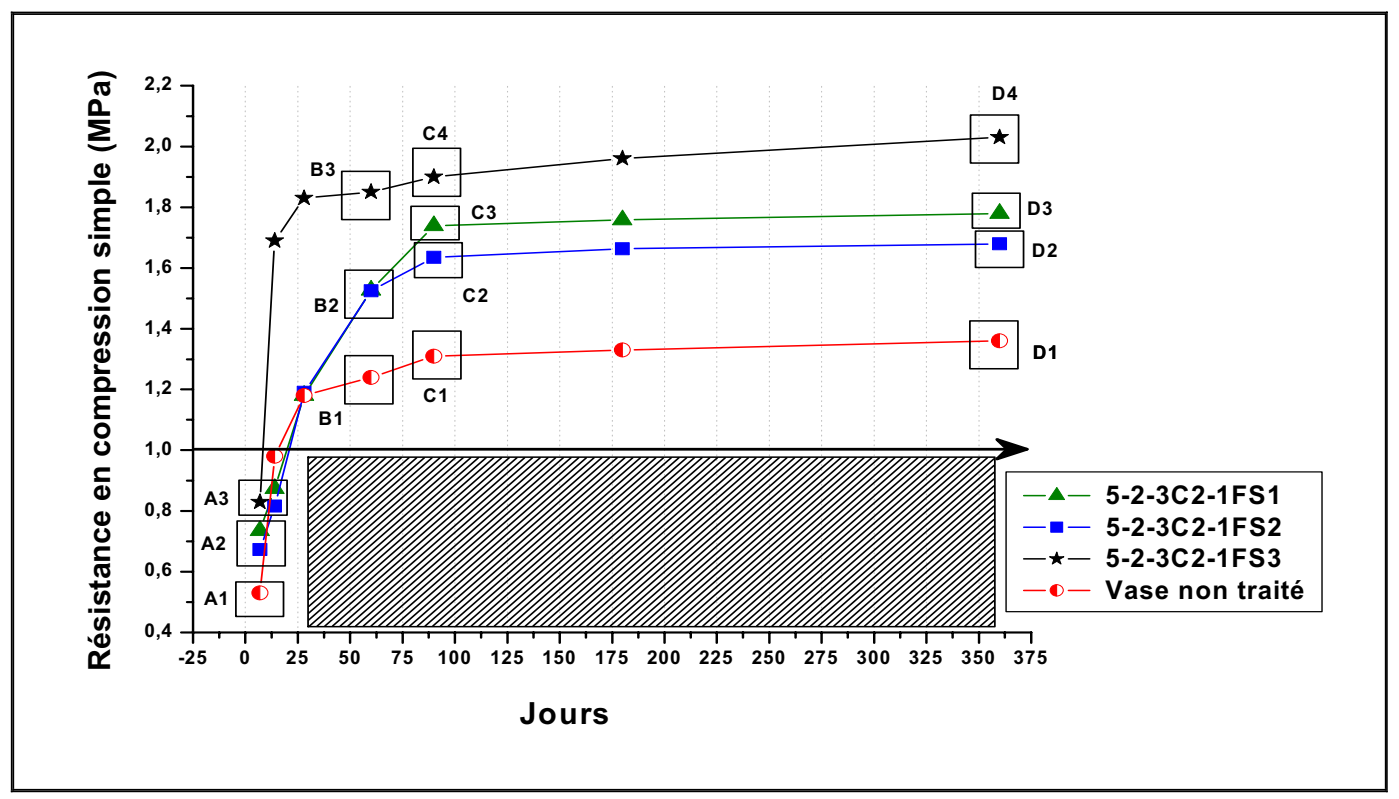

Figure 3. Evolution de Rc pour le sédiment traité avec différents types de fumée de silice avec une addition de $5 \%$ de sable.

Cette augmentation peut être vérifiée depuis l'évolution des performances mécaniques aux jeunes âges, i.e. 7 jours. Sur la figure 3, nous pouvons constater que les résistances 
aux jeunes âges peuvent être différenciées : celle du mélange pour le sédiment non traité (voir A1 sur la figure 3), celle des mélanges traités avec FS1 et FS2 (voir A2 sur la figure 3), et celle du mélange traité avec FS3 (voir A3 sur la figure 3). L'augmentation des résistances aux jeunes âges est théoriquement attribuée à la rapide hydratation du ciment faisant en sorte qu'en augmentant le pourcentage du ciment nous augmentons la performance mécanique du mélange. Donc nous pouvons constater que l'addition de fumée de silice FS3 donne un effet positif sur l'hydratation du ciment. Cet effet dû à FS3 est bien meilleur que pour les autres types de fumées de silice. Nous estimons que cet effet positif de FS3 ne provient pas de sa granulométrie. Nous rappelons que la fumée de silice de type 1 (FS1) contient des particules fines avec des pourcentages plus importants que la fumée de silice type 3 (FS3). La résistance du mélange traité avec FS3 est toujours supérieure, quel que soit l'âge même à long terme (voir C4 et D4 sur la figure 3) avec une valeur de résistance à 360 jours de 2,03 MPa. L'augmentation de la résistance avec $\mathrm{FS} 3$ est de $33 \%$ par rapport au sédiment non traité, elle est respectivement de 23,59\% (FS1) et 19\% (FS2) pour les mélanges traités avec FS1 et FS2.

\subsubsection{Essais de compression simple avec une incorporation de 0 à 15\% de sable}

L'évolution des résistances à 7 jours montre que tous les mélanges ont une valeur presque identique (voir A2 sur la figure 4). A 14 jours, le mélange traité avec FS3 affiche déjà une valeur supérieure aux autres. Ces résultats prouvent encore une fois que la fumée de silice de type 3 (FS3) qui a une réactivité assez importante, joue un rôle et fournit une résistance additionnelle sur le mélange.

Les résistances à 28 jours pour tous les mélanges traités avec la fumée de silice dépassent $1 \mathrm{MPa}$. L'évolution des résistances à 60 jours montre une tendance d'évolution identique à celle des mélanges sans ajout de sable (voir C3 sur la figure 4). Nous pouvons voir que la résistance du mélange traité avec FS1 donne une valeur de 1,9 MPa supérieure aux autres. La figure 4 montre également que c'est le mélange traité avec de la fumée de silice de type 2 (FS2) qui a la plus faible valeur de résistance entre tous les mélanges (voir $\mathrm{C} 2$ sur la figure 4).

Parmi les mélanges traités avec $15 \%$ de sable, en considérant l'évolution de la résistance à long terme (360 jours), c'est le mélange traité avec FS2 qui serait le meilleur comparativement aux autres mélanges (voir D2, D3 et D4 sur la figure 4). Une comparaison de la résistance entre les mélanges traités sans sable, avec 5\% et 15\% de sable est également présentée sur la figure 4. Seuls les mélanges traités avec FS3 sans sable (0-2-3C2-1FS3) et avec 5\% de sable (5-2-3C2-1FS3) ont été retenus parce que ces deux mélanges sont considérés comme étant les meilleurs mélanges (composition). En effet, l'addition de $15 \%$ de sable montre une diminution de résistance depuis l'âge de 7 jours jusqu'à 360 jours, excepté à l'âge 60 jours où le mélange traité avec FS3 et 15\% 
de sable donne une valeur de résistance presque identique à celle des mélanges avec 5\% de sable et sans un ajout de sable (voir C3 sur la figure 4).

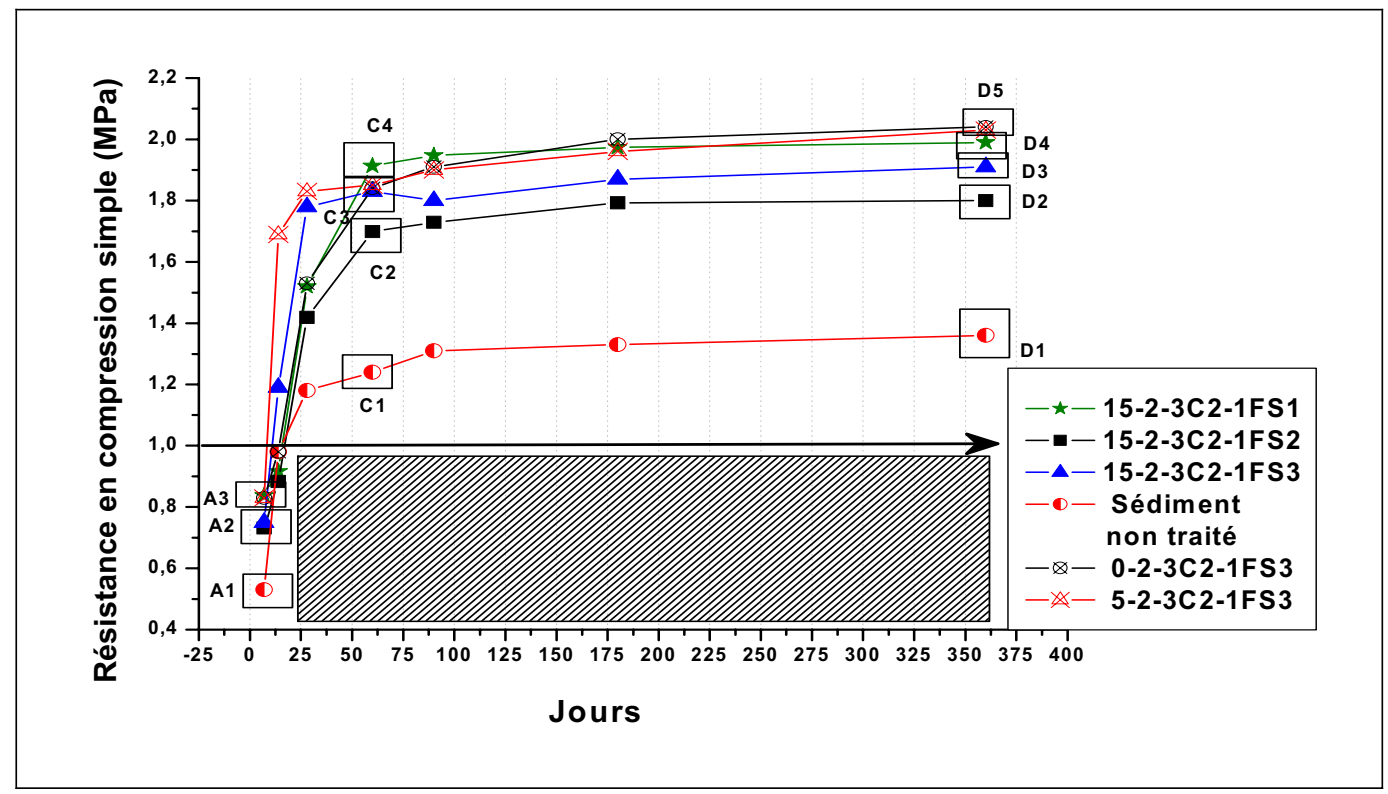

Figure 4. Evolution de Rc pour le sédiment traité avec différents types de fumée de silice avec une addition allant de 0 à $15 \%$ de sable.

3.3 Analyse des résultats des essais de mouillage/séchage

L'essai consiste à comparer les résistances mécaniques en compression simple d'éprouvettes d'âge "j" et ayant subi 10 cycles de mouillage/séchage avec des éprouvettes témoins de même âge et conservées à la température ambiante. Le but des ces essais est d'apprécier la durabilité des échantillons solidifiés lorsqu'ils sont soumis à des cycles répétés de mouillage/séchage. La méthodologie d'essai est tirée et modifiée du protocole d'évaluation proposé pour les déchets solidifiés à base de ciment (STEGEMANN \& COTE, 1992). Chaque cycle de mouillage/séchage commence par une phase de séchage de 24 heures à $60^{\circ} \mathrm{C}$ suivie par une phase de mouillage également fixée à 24 heures et réalisée par immersion totale dans l'eau à température ambiante. Les 10 cycles d'essai sont réalisés sur 20 jours. D'après RAO et al. (2001), les cycles de mouillage-séchage ont un effet négatif sur la durabilité des sédiments stabilisés par la chaux. Il ne faudrait donc pas traiter des sédiments fins à la chaux seule dans les régions qui peuvent subir des cycles mouillage-séchage importants.

\subsubsection{Essais de mouillage/séchage des mélanges sans sable}

Les résultats sont indiqués à la figure 5. L'éprouvette traitée avec de la fumée de silice FS1 montre une résistance presque similaire à l'éprouvette traitée avec de la fumée de silice FS3, avec une valeur de 0,6 MPa. Par contre, pour les éprouvettes de sédiments 
traités avec de la fumée de silice de type FS2, le pourcentage de perte de résistance s'avère le plus important $(71,6 \%)$, avec une résistance obtenue de $0,49 \mathrm{MPa}$. Selon ces résultats, nous pouvons conclure que la fumée de silice de type FS3 présente une moindre perte de résistance aux cycles de mouillage séchage par rapport aux autres types de fumées de silice.

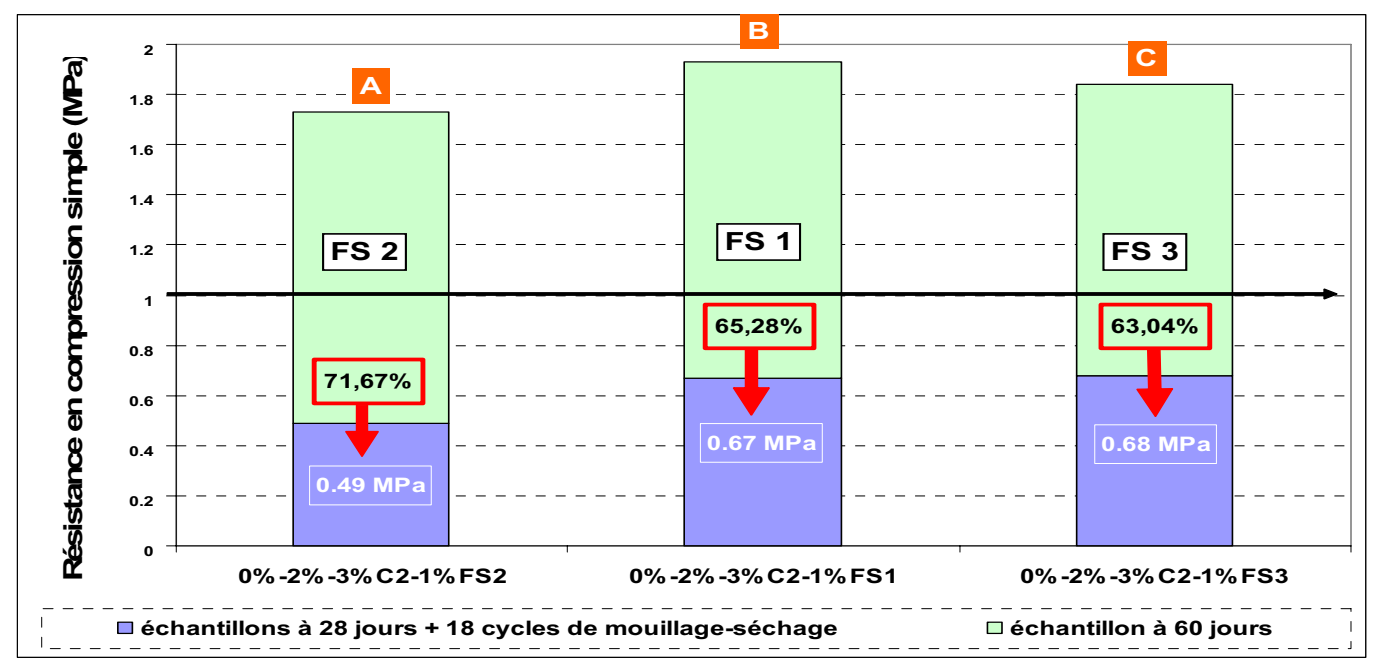

Figure 5. Evolution de la résistance en compression simple d'éprouvettes soumises à des cycles de mouillage-séchage, sans ajout de sable.

\subsubsection{Essais de mouillage/séchage des mélanges avec un ajout de 5\% de sable}

Observons les résultats de la figure 6. Nous estimons que la meilleure formulation d'un mélange avec 5\% de sable, est celle incluant de la fumée de silice type 2 (FS2). Par contre, la meilleure résistance après les essais de mouillage-séchage est celle obtenue avec le mélange traité avec FS3 avec $(0,87 \mathrm{MPa})$.

Nous pouvons également remarquer que l'ajout de $5 \%$ de sable provoque une augmentation assez importante des résistances aux cycles de mouillage-séchage par rapport aux mélanges réalisés sans sable. L'ajout de 5\% de sable donne 21,83\% d'augmentation de la résistance aux cycles de mouillage-séchage pour le mélange traité avec FS3. Ces résultats peuvent confirmer que l'ajout de 5\% de sable fournit une augmentation de la résistance aux cycles de mouillage-séchage très importante pour le mélange traité avec la fumée de silice type 2 (FS2).

\subsubsection{Essais de mouillage/séchage des mélanges traités avec un ajout de15\% de sable}

Dans cette série d'essais, les résultats montrent que le mélange traité avec la fumée de silice de type 3 (FS3) a la perte de résistance la plus faible, égale à $45,23 \%$ (voir A sur la figure 7). Mais la résistance finale au bout de 28 jours et après les cycles de mouillage-séchage pour le mélange traité avec FS1 est la plus élevée ( $0,94 \mathrm{MPa})$, voir C sur la figure 7. 


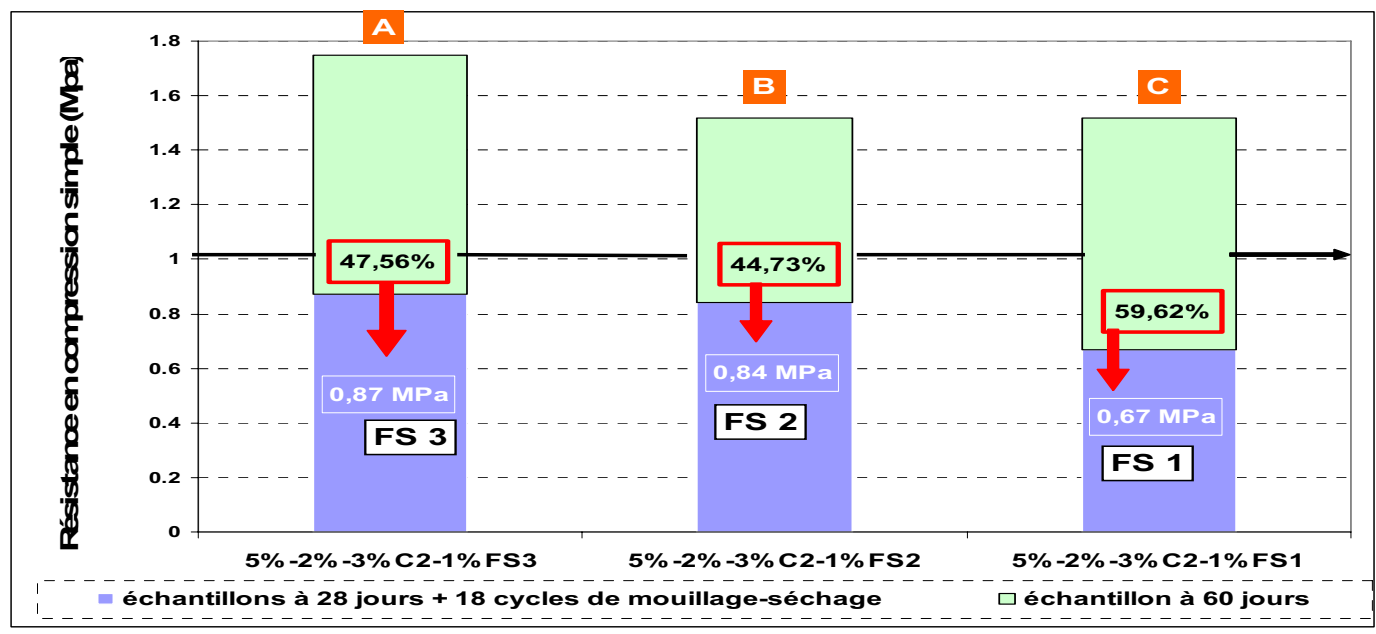

Figure 6. Evolution de la résistance en compression simple d'éprouvettes soumises à des cycles de mouillage-séchage avec 5\% de sable.

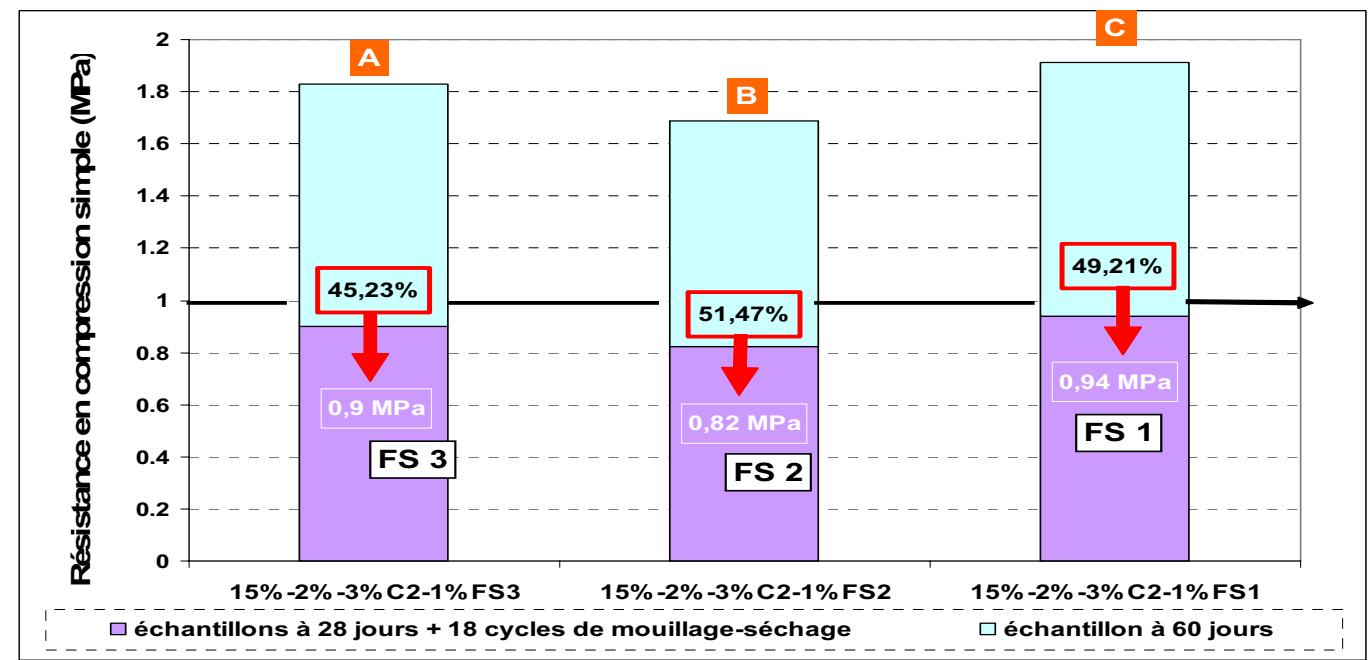

Figure 7. Evolution de la résistance en compression simple d'éprouvettes soumises à des cycles de mouillage-séchage avec 15\% de sable.

Nous estimons que la meilleure formule pour un mélange avec $15 \%$ de sable, est celle établie avec la fumée de silice type 3 (FS3). Les résultats démontent qu'un ajout de 15\% de sables fournit une augmentation de la résistance aux cycles de mouillage-séchage très importante pour le mélange traité avec FS3. En général, l'ajout de 15\% de sable donne une meilleure résistance aux cycles de mouillage-séchage (par rapport aux mélanges avec un ajout de $5 \%$ de sable comme sans sable) pour tous les mélanges sauf pour le mélange traité avec FS2. 


\section{Conclusions}

L'objectif de ce travail et des essais réalisés, consistait à améliorer les propriétés mécaniques et environnementales de sédiments fins dragués de Port-en-Bessin pour une réutilisation en couche de forme ou de fondation routière. Dans ces traitements à base de fumée de silice, différentes formulations en liants ont été effectuées. Le dosage en liant est principalement constitué de $2 \%$ de ciment, de $3 \%$ de chaux, et de $1 \%$ de fumée de silice. Une attention particulière a été portée pour ne pas dépasser un pourcentage maximum de $9 \%$ de liant, taux généralement utilisé dans les travaux routiers et acceptable du point de vue économique. L'effet d'une incorporation de sable comme correcteur de granulométrie a montré un effet irrégulier sur les performances mécaniques des mélanges. Ceci peut être dû à l'incohérence de la granulométrie du sable utilisé. On rappelle que le choix de ce sable était purement économique. La présence d'un liant pouzzolanique (fumée de silice) montre une amélioration des performances mécaniques notamment en résistance à la compression simple. La présence de la fumée de silice montre une amélioration de la résistance en compression simple aux jeunes âges et à long terme. Le mélange traité avec la fumée de silice de type 3 (FS3) sans sable $(0-2-3 \mathrm{C} 2-1 \mathrm{FS} 3)$ et avec 5\% de sable (5-2-3C2-1FS3) ont été finalement retenus parce que ces deux mélanges sont considérés comme étant les meilleurs mélanges.

L'essai de durabilité en conditions extrêmes est étudié au travers l'essai de mouillageséchage. Une addition de $15 \%$ de sable donne une meilleure résistance aux cycles de mouillage-séchage (par rapport aux mélanges avec une incorporation de 5\% de sable ou sans sable). Cette étude complète de caractérisation physico-chimique et géotechnique d'un sédiment de dragage contaminé, en laboratoire en vue d'une valorisation des sédiments, peut servir de modèle pour de futures études à mener dans ce domaine

\section{Références}

ASAVAPISIT S., NANTAMONTRY W., POLPRASERT C. (2001). Influence of condensed silica fume on the properties of cement-based solidified wasted. Cement and Concrete Research, Vol. 31, pp 1147-1152. doi:10.1016//50008-8846(01)00541-5

BAGHERPOUR I., CHOBBASTI A.J. (2003). Stabilization of fine-grained soils by adding microsilica and lime or microsilica and cement. The electronic journal of geotechnical engineering, Volume 8B, 8 p.

BEHMANESH J. (2008). Etude de la durabilité d'un sédiment, traité au ciment et additifs. Thèse de doctorat de l'Université de Caen. $214 \mathrm{p}$.

COCKE D.L., MOLLAH M.Y.A. (1992). The chemistry and microstructure of solidified waste forms. In: R.D. Spense (Ed.), Chemistry and microstructure of solidified waste forms (Ed. by R.D. Spense), pp. 1-39. Lewis.

COCKE D.L., MOLLAH M.Y.A. (1993). The chemistry and leaching mechanisms of hazardous substances in cementious solidification/stabilization systems. In: R.D. 
Spence (Ed.), Chemistry and microstructure of solidified waste forms (Ed. by R.D. Spence), pp 187-242. Lewis.

COCKE D.L., ORTEGO J.D., MCWHINNEY H.G., LEE K., SHULKA S. (1989). $A$ model for lead retardation of cement setting. Cement and concrete research, 19, pp 156-159. doi:10.1016/0008-8846(89)90078-1

GARCIA M.A., CHIMENOS J.M., FERNANDEZ A.I., MIRALLES L., SEGARRA M., ESPEILL F. (2004). Low-grade MgO used stabilize heavy metals in highly contaminated soils. Chemosphere, 56, pp 481-491. doi:10.1016/j.chemosphere.2004.04.005

LEMEE F. (2006). Traitement par alcali activation de sédiments fins marin, non contaminés et à faibles teneur en eau, mise au point d'un procédé de stabilisation. Thèse de doctorat, Université de Caen. $187 \mathrm{p}$.

RAO S.M., REDDY B.V.V., MUTTHARAM M. (2001). The impact of cyclic wetting and drying on the swelling behaviour of stabilized expansive soils. Engineering Geology, Vol. 60, pp 23-233. doi:10.1016/S0013-7952(00)00103-4

STEGEMANN J.A., COTE P.L.(1992). Protocole d'évaluation proposé pour les déchets solidifies a base de ciment. Série de la protection de l’environnement Environnement Canada, $49 \mathrm{p}$. 